\title{
Role of NADPH oxidase and its therapeutic intervention in TGF- $\beta$-mediated EMT progression: an in vitro analysis on HeLa cervical cancer cells
}

\author{
Karthika Muthuramalingam ${ }^{1}$, Moonjae Cho ${ }^{1,2,3^{*}}$ and Youngmee Kim ${ }^{1^{*}}$ (1)
}

\begin{abstract}
Epithelial to mesenchymal transition (EMT) is a complex biological event, wherein polarized epithelial cells lose their integrity resulting in a mesenchymal phenotype with enhanced motility, a phenomenon known as metastasis. However, the underlying mechanisms of EMT are still poorly understood in cervical carcinomas. In this study, we investigated the molecular signalling events responsible for the effect of TGF- $\beta$, a potent inducer of EMT, on HeLa cervical cancer cells. We observed that TGF- $\beta$ treatment $(5 \mathrm{ng} / \mathrm{mL})$ upregulates the expression of EMT-associated transcription factors such as Snail and Slug and downregulates the expression of epithelial markers such as ZO-1 and E-cadherin. Furthermore, treatment with TGF- $\beta$ activates both Smad-dependent and Smad-independent signaling pathways, which subsides upon addition of Diphenyleneiodonium (DPI), a potent ROS inhibitor that inhibits NADPH oxidase (NOX). TGF- $\beta$ treatment enhanced cellular migration and invasion ability was diminished in the presence of ROS inhibitors. In addition, we also observed that ROS-mediated, TGF- $\beta$-induced EMT progression was inhibited using therapeutic candidates that target the key signal transduction mediators, including PI3K/AKT, ERK, and P38/MAPK. Accordingly, we demonstrated the involvement of redox biology (NOX2 and NOX4 mediate migration and invasion) in TGF- $\beta$-mediated EMT advancement and explored suitable therapeutic interventions.
\end{abstract}

Keywords: Cervical cancer, EMT, ROS, TGF- $\beta$, NADPH oxidase

\section{Introduction}

Despite several palliative care and preventive measures such as screening, treatment of pre-cancerous and invasive lesions, and vaccination against the causative agent [Human papilloma virus (HPV) 16 and 18], cervical cancer ranks as the fourth most frequent cancer occurring in women worldwide, with an estimated death total of 311,000 women in 2018 alone [5]. Although numerous studies have been carried out to understand the complex pathways involved in the progression of cervical

\footnotetext{
*Correspondence: moonjcho@jejunu.ac.kr; biochem310@jejunu.ac.kr

${ }^{1}$ Department of Biochemistry, School of Medicine, Jeju National

University, Jeju 63243, Republic of Korea

Full list of author information is available at the end of the article
}

cancer cells, a complete understanding of how this cancer advances is still not available [12]. In order to develop therapeutic candidates to treat cervical cancer, we must first understand the underlying biomolecular signalling mechanisms that induce cancer progression.

Epithelial to mesenchymal transition (EMT) is a complex process in which polarized epithelial cells undergo numerous biological changes, such as spindle-shaped cellular morphology, dissociation of cellular tight junctions, upregulation/downregulation of mesenchymal/ epithelial markers, excessive production of extracellular matrix components, and induction of EMT-associated transcription factors, that result in the acquisition of mesenchymal characteristics $[4,14]$. In the context of physiological tissues, the process of EMT is typically 
divided into three stages. Stage I occurs during embryogenesis and organ development, Stage II occurs during tissue regeneration and the healing process, and Stage III occurs during cancer advancement and metastasis. The phenotypic changes, as well as the invasive and metastatic progression of cancer cells increases drastically during Stage III of EMT [3]. Recent findings support that several EMT-associated regulatory factors are redox sensitive, and thus, explicating the EMT mechanism in the context of redox biology is valuable for exploring the key molecular events underlying cancer progression [6].

Reactive oxygen species (ROS) are involved in several biological events related to cancer cell progression, including cell survival, proliferation, invasion, migration, vascular generation, and metastasis; all of which are also connected with those cells acquiring EMT phenotypic characteristics [17]. Several endogenous (altered P450 metabolism, activation of inflammatory cells, oxidative phosphorylation etc.) and exogenous factors (viral infections, radiation, chemicals, pollutants etc.) contribute to the production of reactive oxygen/nitrogen species [18], which ultimately results in oxidative stress induced damages associated with nucleic acid and protein, thereby leading to cancer malignancy. In cervical cancer, persistent HPV infection and the interaction of HPV oncoprotein components E6 and E7 with tumor suppressor factors and cell cycle regulators lead to the production of ROS followed by cell transformation process [15]. Further numerous antioxidant proteins were found to be deregulated in HPV-associated penile and cervical carcinomas by modulating the accumulation of oxygen species. In addition to viral infections, several other compounds such as TGF- $\beta$ has been shown to induce ROS production and suppress antioxidant system thereby bringing redox imbalance [9]. Despite all these studies, the role of NADPH oxidase (NOX), the major endogenous source of ROS, in cervical cancer progression is not fully elucidated. Thus, in this present study, we planned to study the role of ROS contributor-NADPH oxidase (NOX) and its therapeutic intervention in TGF- $\beta$ mediated EMT progression in HeLa cervical cancer cells. For this, we used an in vitro approach with HeLa cells and the potent EMT inducer TGF- $\beta$ to investigate the redox mechanism behind EMT and its associated cellular events that aids in the progression of cervical cancer.

\section{Materials and methods}

\section{Cell lines and reagents}

The HeLa human cervical cancer cell line (American Type Culture Collection CCL-2), fetal bovine serum (FBS, Gibco Inc., NY, USA), and Dulbecco's Modified Eagle Medium (DMEM, Corning, NY, USA) were used in this study. The chemicals used in this study included transforming growth factor $\beta 1$ (TGF- $\beta 1$ ) (Invitrogen, CA, USA), diphenyleneiodonium (DPI), apocynin (APO), and N-acetyl cysteine (NAC) (Sigma-Aldrich, MO, USA), GKT137831 (Cayman Chemical, Michigan, USA), LY294002 (PI3K inhibitor), U0126 (ERK/MAPK inhibitor), SB203580 (p38/MAPK inhibitor), SB431542 (TGF- $\beta$ type I receptor inhibitor), and PD98050 (ERK inhibitor). The primary antibodies used are as follows: Smad2/3, Smad7, slug, pP38 (Santa Cruz Biotechnology, Dallas, TX. USA), snail, vimentin, AKT, pAKT, p38, ERK, pERK, pSmad2, pSmad3, GAPDH (Cell Signaling Technology, Beverly, MA, USA), NOX4 (Novus Biologicals, Littleton, CO, USA) and ZO-1, NOX2 (BD Biosciences, San Diego, CA, USA).

\section{Cell culture treatment}

HeLa cells were cultured in DMEM supplemented with $10 \%$ FBS and 1\% Penicillin/Streptomycin at standard culture conditions $\left(37{ }^{\circ} \mathrm{C}\right.$ and $5 \% \mathrm{CO}_{2}$ ). After reaching $70 \%$ confluency, the cells were treated with TGF- $\beta$ ( $5 \mathrm{ng} /$ $\mathrm{mL}$ ) for $24 \mathrm{~h}$. For time-course analyses, time points of $0 \mathrm{~h}, 3 \mathrm{~h}, 6 \mathrm{~h}, 12 \mathrm{~h}$, and $24 \mathrm{~h}$ after TGF- $\beta$ treatment were taken into consideration. In order to study the impact of reactive oxygen species (ROS) on factors relating to the epithelial-mesenchymal transition (EMT), experiments were carried out using $1 \mathrm{~h}$ pretreatment with DPI $(5 \mu \mathrm{M})$ followed by $24 \mathrm{~h}$ treatment with TGF- $\beta$. HeLa cells were pretreated for a period of $1 \mathrm{~h}$ with inhibitors such as LY294002 $(50 \mu \mathrm{M})$, U0126 $(40 \mu \mathrm{M})$, SB203580 $(40 \mu \mathrm{M})$, SB431542 $(10 \mu \mathrm{M})$, and PD98050 $(20 \mu \mathrm{M})$ followed by $24 \mathrm{~h}$ treatment with TGF- $\beta$ to analyze the impact of those inhibitors on proteins relating to EMT and NOX.

\section{Migration assay}

Using a $200 \mu \mathrm{L}$ pipette tip, a scratch was made in cell monolayers with more than $80 \%$ confluence. The cell debris formed during the in vitro wounding process was washed away using $1 \mathrm{X}$ phosphate buffer saline (PBS). Furthermore, we pretreated the cells for a period of $1 \mathrm{~h}$ with NAC $(1 \mathrm{mM})$, APO $(10 \mu \mathrm{M})$, DPI $(5 \mu \mathrm{M})$, and GKT $(10 \mu \mathrm{M})$ before incubating the cells with $5 \mathrm{ng} / \mathrm{mL}$ of TGF- $\beta$ for $48 \mathrm{~h}$. Microscopic images [inverted microscope, Olympus CKX41 (Olympus Corporation, Tokyo, Japan)] were taken at $0 \mathrm{~h}$ and $48 \mathrm{~h}$ after TGF- $\beta$ addition and the wound closure was calculated using ImageJ software.

\section{Invasion assay}

Overnight serum-starved (DMEM with 1\% FBS) HeLa cells were seeded onto Transwell inserts with $8 \mu \mathrm{m}$ pore size and immersed in a lower chamber containing DMEM supplemented with $2 \%$ FBS, with or without TGF- $\beta$ ( $5 \mathrm{ng} / \mathrm{mL}$ ) for $48 \mathrm{~h}$, and with $1 \mathrm{~h}$ pretreatment 
Table 1 List of primers used in PCR analysis to detect factors associated with EMT

\begin{tabular}{lll}
\hline Primer & Forward $\left(\mathbf{5}^{\prime} \rightarrow \mathbf{3}^{\prime}\right)$ & Reverse $\left(\mathbf{5}^{\prime} \boldsymbol{\rightarrow} \mathbf{3}^{\prime}\right)$ \\
\hline Snail & GAGGACAGTGGGAAGGCTC & TGGCTTCGCATGTGCATCTT \\
Slug & GAACTCACACGGGGGAGA & ACACAGCAGCCAGATTCCTC \\
& AG & \\
E-cadherin & CCTGGGACTCCACCTACAGA & GGATGACACAGCGTGAGAGA \\
GAPDH & GAGAAGGCTGGGGTCATTT & ACTGATGGCATGGACTGTGG \\
\hline
\end{tabular}

of NAC $(1 \mathrm{mM})$, APO $(10 \mu \mathrm{M})$, DPI $(5 \mu \mathrm{M})$, or GKT $(10 \mu \mathrm{M})$. At the end of the indicated treatment period, non-migrated cells in the top portion of the insert were removed using a cotton swab, while the invaded cells in the bottom portion of the insert were fixed using $4 \%$ paraformaldehyde followed by PBS wash and staining using
$1 \%$ crystal violet solution. The number of cells invaded per field was calculated using ImageJ software.

\section{Western blots}

Proteins were extracted from the treated cells using RIPA buffer and quantified using the Pierce BCA Protein assay kit (Thermo Scientific, MA, USA). Equal amounts of each protein sample were loaded and separated based on their molecular weight using sodium dodecyl sulfate-polyacrylamide gel electrophoresis (SDS-PAGE), followed by transfer to a $0.45 \mu \mathrm{m}$ nitrocellulose blotting membrane for further detection. After blocking the unoccupied sites in the blotting membrane using 10\% skim milk [prepared in 1X Tween 20-Tris buffered saline (TTBS)], the membrane was incubated with primary and secondary antibodies with 4 cycles of 10 min washes using $1 \mathrm{X}$ PBS in

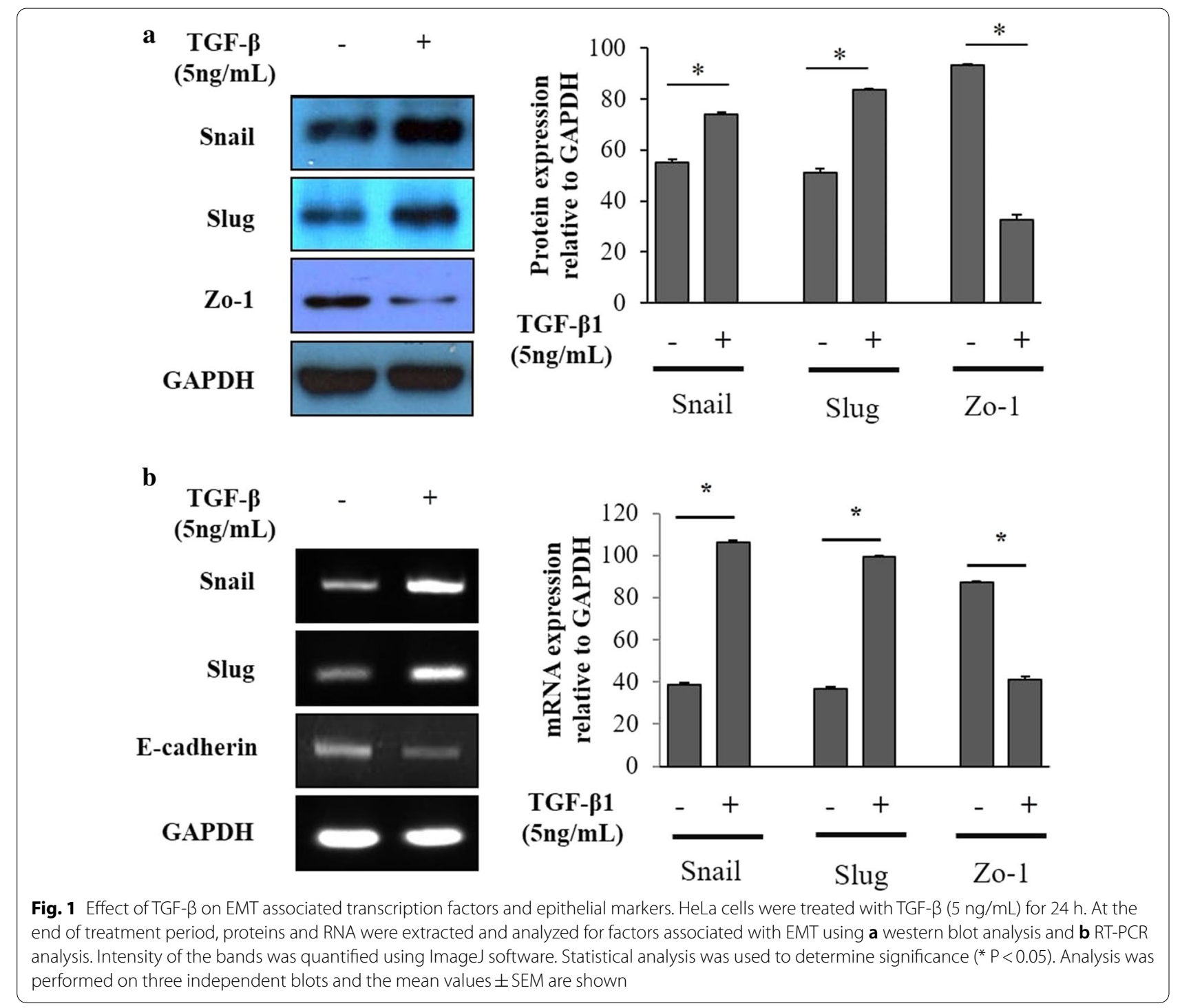


between. The blot was developed using the Western ECL kit (LPS Solution, Daejeon, Republic of Korea) and quantified using ImageJ software.

\section{RT-PCR}

Total RNA isolated from the treated cells [with or without TGF- $\beta$ ( $5 \mathrm{ng} / \mathrm{mL})$ ] using TRIzol reagent (MRC, OH, USA) was converted to complementary DNA (cDNA) using reverse transcription. Equal amounts of the cDNA samples were then used for polymerase chain reactions (PCRs) carried out using the primer sets displayed in Table 1 . The amplified DNA was resolved on a $1 \%$ agarose gel containing TopRed nucleic acid staining solution (Biopure, UK). The intensity of the bands was quantified using ImageJ software.

\section{Statistical analysis}

All data are represented as mean \pm standard error of the mean. Statistical significance was analyzed using t-tests and the value was considered to be significant when $\mathrm{P}<0.05$.

\section{Results and discussion}

As principal cellular secondary messengers, reactive oxygen species (ROS) are involved in a myriad of biological functions including the initiation and advancement of epithelial to mesenchymal transition (EMT) by modulating extracellular matrix (ECM) components, cell-contact junctions, and metastasis induction [17]. However, the precise molecular mechanism and key modulators underlying the involvement of ROS in EMT and cancer progression is still poorly understood. In this study, we investigated the impact of ROS on cell migration and EMT upon induction with TGF- $\beta$.

We observed that transcription factors Snail and Slug, which are responsible for upregulating and downregulating genes relating to the mesenchymal and epithelial phenotypes, were found to be more highly expressed in HeLa cells treated with TGF- $\beta$ than in the untreated control cells at both the translational (Fig. 1a) and transcriptional (Fig. 1b) levels. Furthermore, both ZO-1 (tight junction-associated marker) and E-cadherin (adherent junction-associated marker), which relate to the epithelial phenotype, were found to be significantly reduced in the TGF- $\beta$ treated group compared to that of the control group. These data indicate that EMT is favored during the addition of TGF- $\beta$.

Next, we wanted to observe the dynamics of EMT in HeLa cells treated with TGF- $\beta$ because EMT is associated with temporal changes in the cell polarity, cell-cell junction, cytoskeletal rearrangement, and stress fiber formation. We detected a time-dependent increase in the expression of Snail (Fig. 2). In addition, we also measured an increase in the expression of activated Smad proteins (pSmad2 and $\mathrm{pSmad} 3$ ) upon TGF- $\beta$ treatment (Fig. 2). After TGF- $\beta$ treatment, non-canonical TGF- $\beta$ / ERK activation peaked at $3 \mathrm{~h}$ and gradually subsided over time, only to get reactivated to a greater intensity at 24 h. Furthermore, phosphorylated P38/MAPK and activated AKT [phospho AKT (Ser473)] proteins were highly expressed in the early and late time points of TGF- $\beta$ treatment, respectively. Apart from enhancing cellular mobility, the activated PI3K/AKT pathway contributes towards the disruption of EMT-linked tight junction proteins [16], which is supported by the decrease in the expression of ZO-1 and E-cadherin proteins that we observed in the HeLa cells treated with TGF- $\beta$ (Fig. 1a, b). Phosphorylated P38/MAPK levels are an indicator of mesenchymal phenotype acquisition by the cells, with higher levels corresponding to less epithelial cell characteristics [2]. Activation of ERK is involved in the induction of EMT-associated transcription factors (such as Snail and Twist) along with the transcription of matrix metalloproteins (MMPs), which are responsible for ECM stabilization and thereby enhancing EMT progression $[10,13]$. Therefore, these data indicate the involvement of

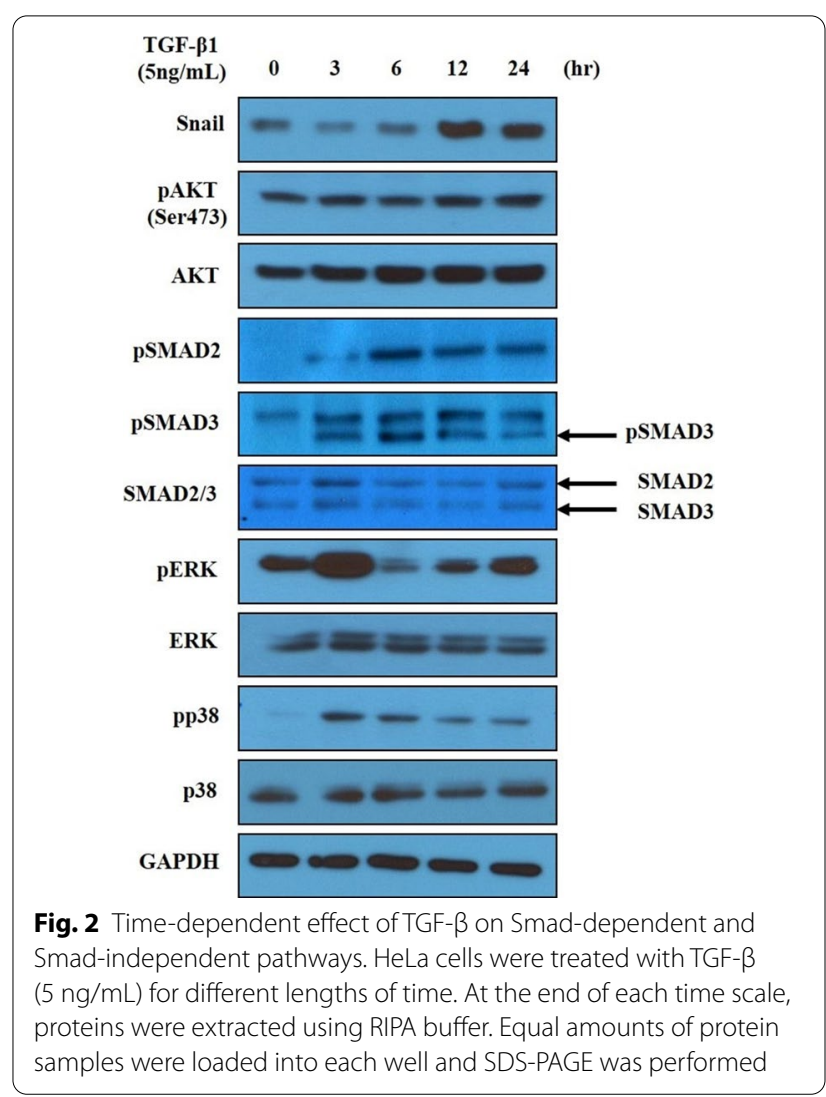


canonical (Smad-dependent) and non-canonical (Smadindependent) pathways in the dynamics of TGF- $\beta$ signaling towards EMT advancement.

Reactive oxygen species (ROS) are involved with several biological events related to cancer cells, such as cell survival, proliferation, invasion, migration, vascular generation, and metastasis [6]. All of these biological events are also connected with those cells acquiring EMT phenotypic characteristics. Therefore, we next investigated whether the already observed TGF- $\beta$-induced EMT effect is linked to ROS or not. Apart from exogenous factors such as chemicals, chemotherapy, radiation, and pollutants, several endogenous factors, including the mitochondrial respiratory chain complex, NADPH oxidase (NOX), dual oxidase (DUOX), and oxygenase, exist as sources of ROS in the cell [8]. We treated HeLa cells with diphenyleneiodonium (DPI), an inhibitor of NOX, along with TGF- $\beta$ and observed a decrease in the expression of TGF- $\beta$-induced, EMT-associated, non-canonical signaling proteins pAKT (Ser473), pP38, and pERK, thereby establishing the link between ROS and EMT progression (Fig. 3). Upon treating the cells with DPI (NOX inhibitor and antioxidant), NAC (ROS scavenger), APO
(NOX inhibitor and antioxidant), and GKT (NOX4/ NOX1 inhibitor), we observed that the TGF- $\beta$-induced cellular migration (Fig. 4a) and invasion ability (Fig. 4b) of the HeLa cells was significantly diminished. The difference in effect imposed by the above-mentioned therapeutic agents might be attributed to the disparity in their mode of action in inhibiting NOX isoforms and other endogenous sources of ROS as follows: While NAC being radical scavenger through aiding in the synthesis of glutathione (GSH), a powerful antioxidant [1], GKT acts as specific NOX1/4 inhibitor [11]. Apocynin and DPI is said to non-specifically inhibit ROS production from diverge sources (such as mitochondrial ROS production, cytochrome P450 mono-oxygenase, Nitric oxide synthase, Xanthine oxidase, NOX enzymes and other flavoproteins). Further, Apocynin and DPI is said to exhibit pro-oxidant activity in some instances thereby unexpectedly imposing oxidative stress to the cellular system instead of recovering the cells from enhanced intracellular ROS impact [7].

With the observed connection between EMT and ROS, we wanted to further analyze the impact of tumorigenic signaling cascade mediators on ROS generation.

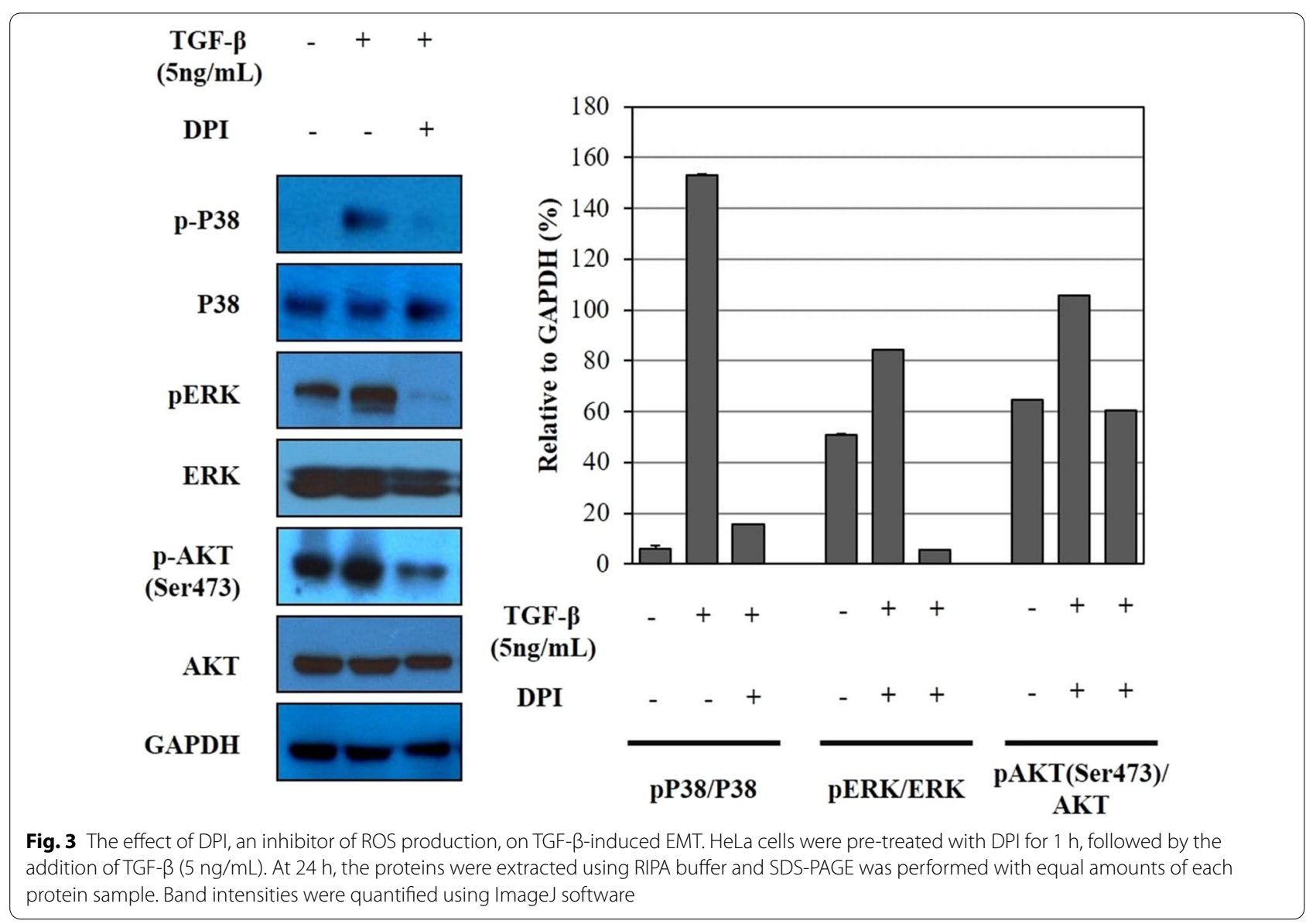



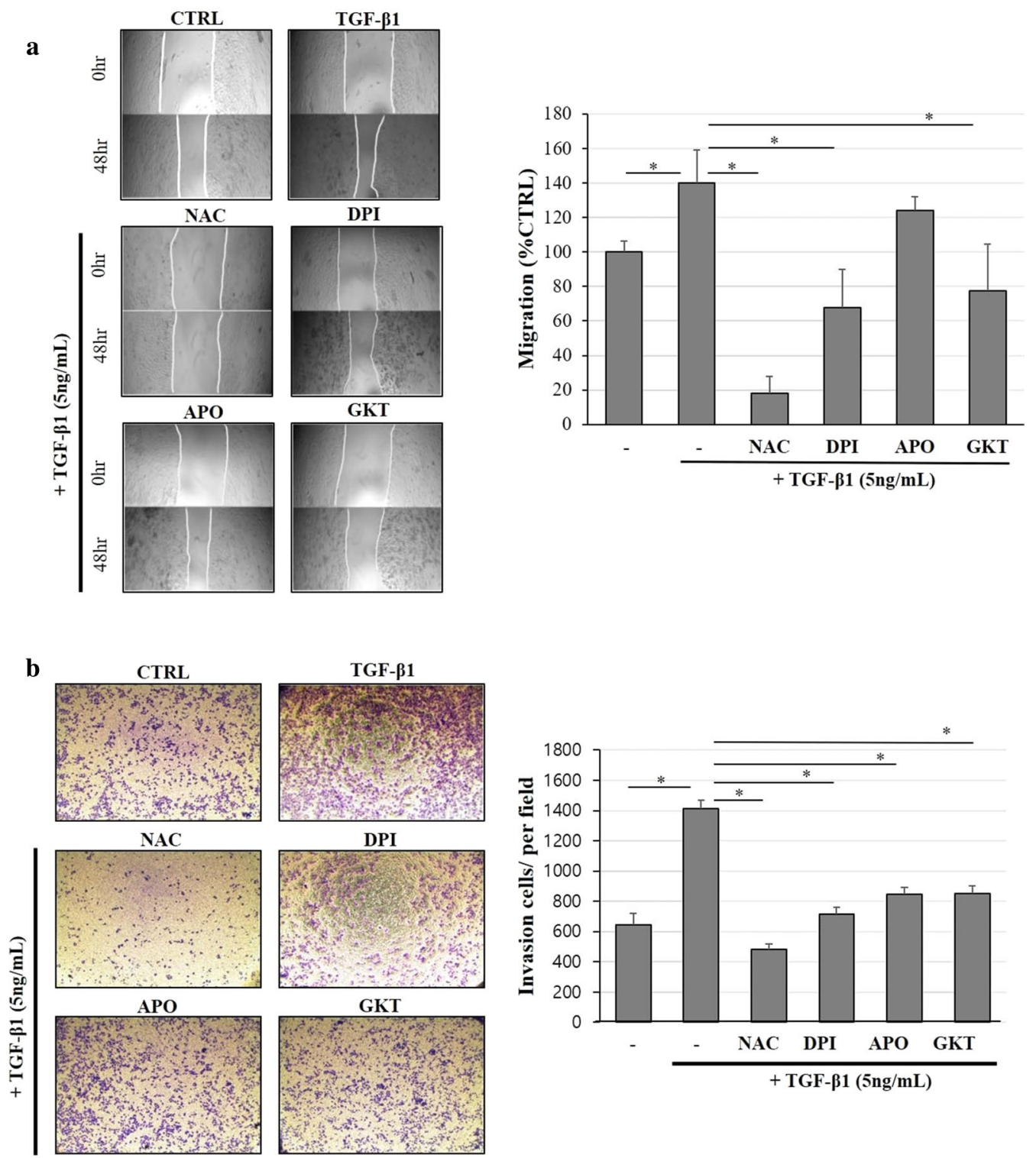

Fig. 4 The effect of ROS inhibitors on TGF- $\beta$ mediated cell migration and invasion ability of HeLa cells. HeLa cells were treated for $48 \mathrm{~h}$ with the ROS inhibitors DPI, NAC, APO, and GKT along with TGF- $\beta(5 \mathrm{ng} / \mathrm{mL})$. a The migration capacity was analyzed using scratch wound healing assays and b the invasion ability was analyzed using Transwell migration assays. The amount of wound closure and cell invasion was calculated using ImageJ software analysis. Statistical analysis was used to determine if treatments were significant $\left({ }^{*} P<0.05\right)$. Analysis was performed on three independent blots and the mean values \pm SEM are shown

During TGF- $\beta$ treatment of HeLa cells, increased NOX expression (NOX4 and NOX2) was drastically hindered with the addition of inhibitors that targeted tumorigenic mediators such as TGF- $\beta$, AKT, ERK, and P38 (Fig. 5a). At this stage of the current research, we could only able to hypothesis that there exists a feed forward loop between NOX activation and stress stimuli such as AKT, ERK and P38MAPK. Thus, NOX induced stress stimuli when inhibited downregulates NOX. One possible mechanism behind this observation could be of inflammatory cytokine mediators. Upon treating the cells with TGF-beta, NOX gets induced thereby activating its downstream effector molecules such as P38MAPK and ERK, which in turn is expected to trigger inflammatory cytokine molecules and thus ROS. This redox imbalance is said to activate TGF-beta thereby triggering further NOX induction. Thus, inhibiting the stress stimuli such as P38MAPK and ERK downregulated NOX expression 


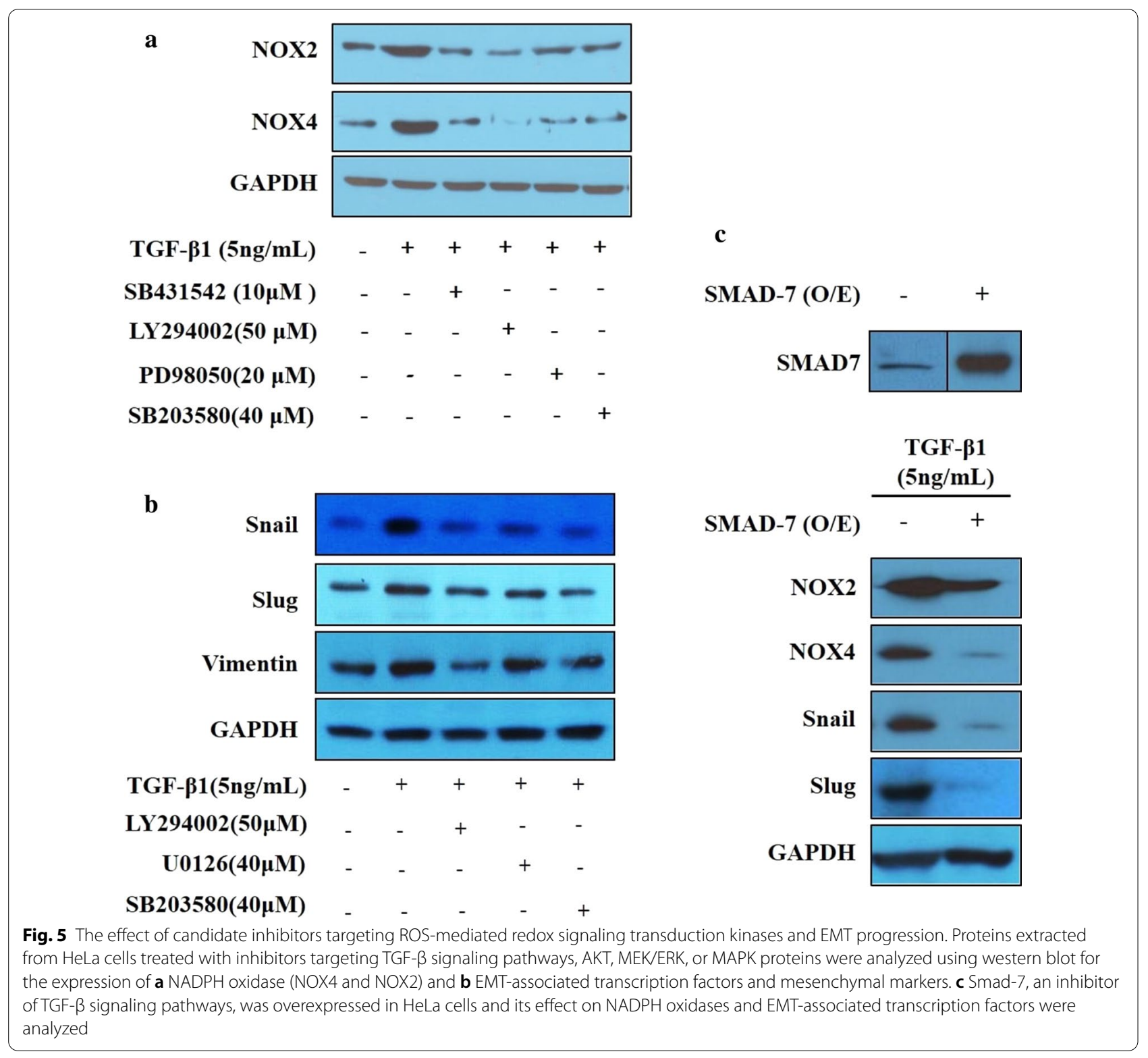

in our study. In addition, upon using these inhibitors, the expression of EMT-related proteins Snail, Slug, and Vimentin were reduced (Fig. 5b). Ectopic expression of Smad7, a TGF- $\beta$ inhibitor, repressed TGF- $\beta$ mediated EMT signaling and NOX expression (Fig. $5 \mathrm{c}$ ), presumably by interacting with activated TGF- $\beta$ receptors and thereby blocking nuclear translocation of the Smad2/3 complex and abrogating EMT-associated gene transcription.

Altogether, these results suggest that EMT and its underlying processes, such as migration and invasion, are mediated by NOX enzymes (NOX2 and NOX4). Drug candidates targeting NOX induction and redox-signal transduction cascade kinases inhibit NOX-mediated EMT progression thereby exhibiting promising results against TGF- $\beta$ mediated cervical cancer advancement.

\section{Abbreviations}

APO: apocynin; DPI: diphenyleneiodonium; DUOX: dual oxidase; ECM: extra cellular matrix; EMT: epithelial to mesenchymal transition; HPV: human papilloma virus; MMP: matrix metalloprotein; NAC: N-acetyl cysteine; NOX: NADPH oxidase; ROS: reactive oxygen species; TGF- $\beta$ : transforming growth factor $\beta$.

\section{Authors' contributions}

YMK performed the experiments. KM assisted YMK in result analysis and wrote the manuscript. YMK and MJC conceived and designed the experiments along with correction of the manuscript. All authors read and approved the final manuscript. 


\section{Funding}

This research was supported by the Basic Science Research Program through the National Research Foundation of Korea (NRF) funded by the Ministry of Education, Science and Technology (NRF-2017R1C1B2011197).

\section{Availability of data and materials}

Not applicable.

\section{Compliance with ethical standards}

\section{Competing interests}

The authors declare that they have no competing interests.

\section{Author details}

${ }^{1}$ Department of Biochemistry, School of Medicine, Jeju National University, Jeju 63243, Republic of Korea. ${ }^{2}$ Institute of Medical Science, Jeju National University, Jeju 63241, Republic of Korea. ${ }^{3}$ Interdisciplinary Graduate Program in Advanced Convergence Technology \& Science, Jeju National University, Jeju 63241, Republic of Korea.

Received: 10 November 2019 Accepted: 21 December 2019 Published online: 09 January 2020

\section{References}

1. Aldini G, Altomare A, Baron G et al (2018) N-Acetylcysteine as an antioxidant and disulphide breaking agent: the reasons why. Free Radical Res 52:751-762. https://doi.org/10.1080/10715762.2018.1468564

2. Cheng HH, Chu LY, Chiang LY et al (2016) Inhibition of cancer cell epithelial mesenchymal transition by normal fibroblasts via production of 5-methoxytryptophan. Oncotarget 7:31243-31256. https://doi. org/10.18632/oncotarget.9111

3. Giannoni E, Parri M, Chiarugi P (2012) EMT and oxidative stress: a bidirectional interplay affecting tumor malignancy. Antioxid Redox Signal 16:1248-1263. https://doi.org/10.1089/ars.2011.4280

4. Hiraga R, Kato M, Miyagawa S, Kamata T (2013) Nox4-derived ROS signaling contributes to TGF- $\beta$-induced epithelial-mesenchymal transition in pancreatic cancer cells. Anticancer Res 33:4431-4438

5. Jaiswal A, Sabarwal A, Narayan Mishra JP, Singh RP (2018) Plumbagin induces ROS-mediated apoptosis and cell cycle arrest and inhibits EMT in human cervical carcinoma cells. RSC Adv 8:32022-32037. https://doi. org/10.1039/c8ra05339a

6. Jiang J, Wang K, Chen Y et al (2017) Redox regulation in tumor cell epithelial-mesenchymal transition: molecular basis and therapeutic strategy. Signal Transduct Targ Ther 2:1-12. https://doi.org/10.1038/sigtr ans.2017.36

7. Kučera J, Binó L, Štefková K et al (2016) Apocynin and diphenyleneiodonium induce oxidative stress and modulate PI3K/Akt and MAPK/Erk activity in mouse embryonic stem cells. Oxid Med Cell Longev. https:// doi.org/10.1155/2016/7409196

8. Lee BWL, Ghode P, Ong DST (2018) Redox regulation of cell state and fate. Redox Biol. https://doi.org/10.1016/j.redox.2018.11.014

9. Liu RM, Desai LP (2015) Reciprocal regulation of TGF- $\beta$ and reactive oxygen species: a perverse cycle for fibrosis. Redox Biol 6:565-577. https ://doi.org/10.1016/j.redox.2015.09.009

10. Moustakas A, Heldin P (2014) TGF $\beta$ and matrix-regulated epithelial to mesenchymal transition. Biochim Biophys Acta Gen Subj 1840:26212634. https://doi.org/10.1016/j.bbagen.2014.02.004

11. Murphy-Marshman H, Quensel K, Xu SW et al (2017) Antioxidants and NOX1/NOX4 inhibition blocks TGF 31 -induced CCN2 and a-SMA expression in dermal and gingival fibroblasts. PLOS ONE 12:1-15. https://doi. org/10.1371/journal.pone.0186740

12. Qureshi R, Arora H, Rizvi MA (2015) EMT in cervical cancer: Its role in tumour progression and response to therapy. Cancer Lett 356:321-331. https://doi.org/10.1016/j.canlet.2014.09.021

13. Scott LE, Weinberg SH, Lemmon CA (2019) Mechanochemical signaling of the extracellular matrix in epithelial-mesenchymal transition. Front Cell Dev Biol. https://doi.org/10.3389/fcell.2019.00135

14. Serrano-Gomez SJ, Maziveyi M, Alahari SK (2016) Regulation of epithelialmesenchymal transition through epigenetic and post-translational modifications. Mol Cancer 15:1-14. https://doi.org/10.1186/s12943-016-0502-x

15. Silva GÁF, Nunes RAL, Morale MG et al (2018) Oxidative stress: therapeutic approaches for cervical cancer treatment. Clinics (Sao Paulo, Brazil) 73:1-10. https://doi.org/10.6061/clinics/2018/e548s

16. Vignais M, Fafet P. TGFß-dependent Epithelial-Mesenchymal Transition. In: Madame Curie Bioscience Database [Internet]. Austin (TX): Landes Bioscience; 2000-2013. https://www.ncbi.nlm.nih.gov/books/NBK6525/

17. Wang Z, Li Y, Sarkar F (2010) Signaling mechanism(s) of reactive oxygen species in epithelial-mesenchymal transition reminiscent of cancer stem cells in tumor progression. Curr Stem Cell Res Ther 5:74-80. https://doi. org/10.2174/157488810790442813

18. Zhang J, Wang X, Vikash V et al (2016) ROS and ROS-mediated cellular signaling. Oxid Med Cell Longev. https://doi.org/10.1155/2016/4350965

\section{Publisher's Note}

Springer Nature remains neutral with regard to jurisdictional claims in published maps and institutional affiliations.

\section{Submit your manuscript to a SpringerOpen ${ }^{\circ}$ journal and benefit from:}

- Convenient online submission

- Rigorous peer review

- Open access: articles freely available online

- High visibility within the field

- Retaining the copyright to your article

Submit your next manuscript at $\mathbf{s p r i n g e r o p e n . c o m ~}$ 